\title{
Effect of Stress on Track Formation in Amorphous Iron Boron Alloy: Ion Tracks as Elastic Inclusions
}

\author{
C. Trautmann, ${ }^{1}$ S. Klaumünzer, ${ }^{2}$ and H. Trinkaus ${ }^{3, *}$ \\ ${ }^{1}$ Gesellschaft für Schwerionenforschung, Planckstrasse 1, D-64291 Darmstadt, Germany \\ ${ }^{2}$ Hahn Meitner Institut, Postfach 390128, D-14091 Berlin 39, Germany \\ ${ }^{3}$ Institut für Festkörperforschung, Forschungszentrum Jülich, D-52425 Jülich, Germany
}

(Received 6 June 2000)

\begin{abstract}
In a recently developed model of ion beam induced plastic deformation of amorphous solids, ion tracks are described as cylindrical thermoelastic inclusions formed upon local heating and shear stress relaxation along the ion trajectories. According to this model, track formation can be influenced or even suppressed by an applied stress. This model prediction is tested by studying the influence of stress on the etching of tracks of $2.4 \mathrm{GeV} \mathrm{Pb}$ in foil samples of the glassy metal $\mathrm{Fe}_{81} \mathrm{~B}_{13.5} \mathrm{Si}_{3.5} \mathrm{C}_{2}$, where a compressive in-plane stress was built up in limited zones by preirradiation with a high fluence of $200 \mathrm{MeV} \mathrm{Xe}$ ions. The variation of the size of the observed etch pits with the local stress is found to be consistent with the model predictions, thus confirming the thermal spike origin of the tracks.
\end{abstract}

PACS numbers: 61.43.Dq, 61.80.Az, 61.80.Jh

Energetic ions penetrating solids are slowed down by their interaction with the target nuclei (nuclear energy loss) and with the target electrons (electronic energy loss). The latter interaction dominates at high kinetic energies $\left(E_{\mathrm{kin}} \geq 1 \mathrm{MeV}\right.$ per nucleon) where the resulting electronic excitation energy density can be so large that each ion creates a latent track of modified target material along its trajectory [1-3]. Such tracks are also denoted as linear or columnar defects because of typical lengths of tens of micrometers contrasting with comparatively tiny widths of several nanometers. They have been observed to form in many crystalline solids, particularly in insulators, but also in various amorphous materials [4-9] where they are associated with irreversible macroscopic deformation [10-14]. Properties of ion tracks in insulators, semiconductors, high- $T_{c}$ superconductors, and polymers are exploited in both fundamental and applied sciences as well as microtechnology [1-3].

The classical method to reveal latent ion tracks in solids is chemical etching [2,3]. Successful track etching requires the etching velocity to be higher along the track than on the surface of the surrounding sample material. From a chemical point of view, this increase of the etching velocity is due to an increase of the chemical potential of the material in the track region.

In crystalline solids (particularly in insulators), ion tracks are frequently found to be in the amorphous state. In such cases, the difference between the chemical potentials in the track and its surroundings, $\delta \mu_{t}$, is that between the amorphous and the crystalline state which is of the order of the thermal energy of an atom at the melting temperature, $k T_{m}$, i.e., typically some tenths of $\mathrm{eV} /$ atom. In amorphous materials where tracks are in some kind of modified amorphous state, the situation is less clear but $\delta \mu_{t}$ is certainly substantially smaller than for tracks in crystalline materials. In this Letter, we are considering the only amorphous metallic alloy
$\mathrm{Fe}_{81} \mathrm{~B}_{13.5} \mathrm{Si}_{3.5} \mathrm{C}_{2}$ for which track etching has been successfully demonstrated so far [5]. For this system, we estimate $\delta \mu_{t}$ on the basis of the following simple defect picture. Since the metallic character of this alloy ensures a rapid decay of irradiation induced defects in the electronic subsystem, only structural defects resulting from atomic displacements need to be considered. According to Egami and Srolovitz [15] the value for the formation enthalpy of defects of Frenkel pair type in amorphous iron can be estimated to be about $0.6 \mathrm{eV}$. Low-temperature irradiation of amorphous $\mathrm{Fe}_{75} \mathrm{~B}_{25}$ and subsequent annealing yields a maximum atomic concentration of such defects of less than $1 \times 10^{-3}$ at room temperature [16]. Hence, for the metallic glass under consideration we find an upper bound estimate for the defect contribution to $\delta \mu_{t}$ of $\delta \mu_{t}^{\text {def }}$ $(T \approx 300 \mathrm{~K})<0.6 \mathrm{meV} /$ atom which is far below $k T$ at room temperature.

In view of these considerations, the question arises whether structural defects provide the only contribution to track etching in amorphous materials or whether there is some other, possibly more important type of contribution. In fact, a model [17-20] recently developed to rationalize the deformation behavior of amorphous materials under bombardment with track-generating ions [10-14] predicts the existence of a significant elastic contribution to $\delta \mu_{t}$. It is the purpose of this Letter to test this prediction by appropriately designed etching experiments.

In this model, the energy initially deposited into the electronic subsystem is assumed to be rapidly $\left(<10^{-14} \mathrm{~s}\right)$ randomized and subsequently transferred to the atomic subsystem. At times larger than $5 \times 10^{-13} \mathrm{~s}$ the overwhelming part of this energy appears as heat accompanied by thermoelastic stresses. At a sufficiently large electronic excitation energy density and sufficiently strong coupling between both subsystems, local heating leads to melting and, consequently, to shear stress relaxation. Subsequent freezing-in of the strain increments associated with this 
shear stress relaxation in the melt results in a permanent contribution to macroscopic deformation. The superposition of these strain increments can quantitatively describe the experimentally observed macroscopic deformation phenomena in the form of anisotropic growth (extension perpendicular to the ion beam) [10-12] and creep under an externally applied stress [12-14].

According to the scenario considered in this type of "thermal spike model," a frozen ion track in an amorphous material represents a cylindrical mesoscopic defect in the form of a strained (thermo-)elastic inclusion. Depending on the thermal volume expansion coefficient $\alpha$ and the effective freezing temperature $T^{*}$, the elastic energy density stored in a track in an otherwise stress-free sample can yield a significant contribution $\delta \mu_{t}^{\mathrm{el}}$ to the increase in the chemical potential $\delta \mu_{t}$. Using $\alpha=4.5 \times 10^{-5} \mathrm{~K}^{-1}$ and $T^{*}=1020 \mathrm{~K}=0.8 T_{m}$ for $\mathrm{Fe}_{81} \mathrm{~B}_{13.5} \mathrm{Si}_{3.5} \mathrm{C}_{2}$ in the relevant theoretical expressions of the thermoelastic model [19], we obtain $\delta \mu_{t}^{\mathrm{el}}=0.8 \mathrm{meV} /$ atom, i.e., a value comparable or even above the contribution of defects to $\delta \mu_{t}$. Hence, the elastic energy stored in the track must be considered to affect track etching. We emphasize in this context that track revelation in such amorphous systems requires cautiously performed soft etching procedures since the difference in the chemical potential between the etchant and the sample material without tracks, $\Delta \mu_{b}$, must not be substantially larger than the rather small values expected for $\delta \mu_{t}$.

An important prediction of the model suited for testing its basic ideas is that it should be possible to tune the stress state of a track by an externally applied stress or some large-scale internal stress. In more detail, an appropriately chosen stress should be able to partly or fully compensate the shear stress (nonhydrostatic part of the stress tensor) induced by the thermal expansion in a nascent track thus preventing shear stress relaxation during the hot phase of the thermal spike and, correspondingly, the formation of a thermoelastic inclusion during its cooling-down phase. Hence, it should be possible to vary the etchability of ion tracks in amorphous materials systematically by applying mechanical stresses.

We have tested this prediction by experiments on $\mathrm{Fe}_{81} \mathrm{~B}_{13.5} \mathrm{Si}_{3.5} \mathrm{C}_{2}$ samples. 32- $\mu \mathrm{m}$-thick foils of this material were cut into small pieces of $3 \times 20 \mathrm{~mm}^{2}$. Selected areas of $3 \times 2 \mathrm{~mm}^{2}$ (regions 1 and $1^{\prime}$ in Fig. 1) were preirradiated with $200 \mathrm{MeV}{ }^{129} \mathrm{Xe}$ ions at normal beam incidence up to $5.6 \times 10^{17}$ ions $/ \mathrm{m}^{2}$ at about $80 \mathrm{~K}$ at the cyclotron of the Ionenstrahllabor Berlin. The other sample parts (regions 2 and $2^{\prime}$ in Fig. 1) were covered by thick copper masks. According to the TRIM95 code [21], the projected range $R_{p}$ of $200 \mathrm{MeV}$ Xe ions in $\mathrm{Fe}_{81} \mathrm{~B}_{13.5} \mathrm{Si}_{3.5} \mathrm{C}_{2}$ is about $9.8 \mu \mathrm{m}$ and thus considerably less than the specimen thickness. During preirradiation the irreversible anisotropic deformation process [10-12] in region 1 in combination with the mechanical constraint of the unirradiated bottom layer results in the buildup of a compressive in-plane stress. The fluence of $5.6 \times 10^{17}$ ions $/ \mathrm{m}^{2}$ is large enough to ensure that the compressive saturation stress of about $2 \mathrm{GPa}$ is reached from the surface to a depth of about 5 to $6 \mu \mathrm{m}[12,14]$ which is expected to prevent the further formation of strained ion tracks. In regions $1^{\prime}$, the irradiated material is not fully constrained by surrounding material and plastic flow towards the free edges results in a continuous decrease of the magnitude of the stress towards the free edges. In region $2^{\prime}$ adjacent to region 1, elastic equilibrium requires a continuous decay of the stress field within a scale of the order of micrometers. In region 1, below the depth of 5 to $6 \mu \mathrm{m}$, the stress is essentially zero up to a depth of $R_{p}$. In the unirradiated region 3 below $R_{p}$, the in-plane compressive stress of region 1 is balanced by an in-plane tensile stress which is expected to assist track formation.

After the preirradiation with Xe ions, the samples were exposed to a $\mathrm{Pb}$ ion beam of $2.4 \mathrm{GeV}$ at the linear accelerator UNILAC of the GSI in Darmstadt. Note that in this second irradiation all parts of the specimens, i.e., regions $1,1^{\prime}$, 2 , and $2^{\prime}$, were homogeneously bombarded at room temperature. The applied fluence of $7 \times 10^{10}$ ions $/ \mathrm{m}^{2}$ was low enough to study individual tracks and large enough to study spatial changes in their etchability. The $2.4-\mathrm{GeV} \mathrm{Pb}$ ions pass through the samples with almost unaltered electronic energy loss of $55 \mathrm{keV} / \mathrm{nm}$ which is well above the etching threshold of $34 \mathrm{keV} / \mathrm{nm}[6,7]$.

Track etching was performed at room temperature in a $0.7-\mathrm{N}$ solution of $\mathrm{HCl}$ which was immersed into an ultrasonic bath. During the etching process of $1 \mathrm{~min}$ a dc voltage of $1 \mathrm{~V}$ was applied using the sample as anode and a platinum foil as cathode. As expected under the given etching conditions, conical etch pits with wide opening angles of about $90^{\circ}$ and diameters between 2.5 and $3 \mu \mathrm{m}$ were identified in region 2. Regular etch pits of comparable size were also found on the entire backside of the sample including region 3 . The number of pores corresponds to the applied ion fluence. Scanning from region 2

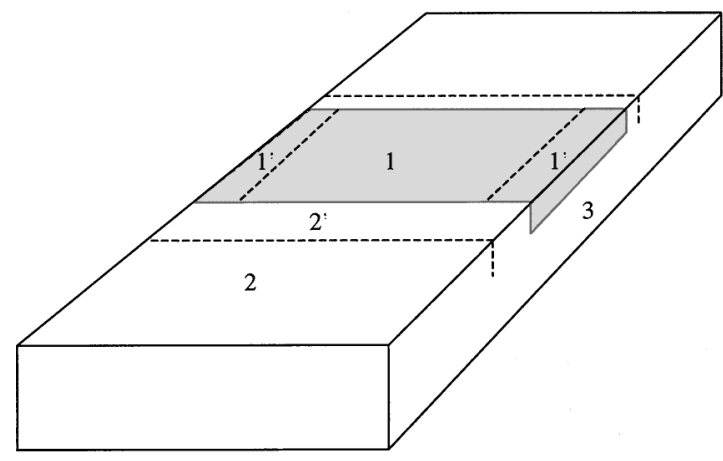

FIG. 1. Geometry of the $32-\mu$ m-thick $\mathrm{Fe}_{81} \mathrm{~B}_{13.5} \mathrm{Si}_{3.5} \mathrm{C}_{2}$ sample: compressive in-plane stress in region 1 induced by high fluence irradiation with $5.6 \times 10^{17} \mathrm{Xe}$ ions $/ \mathrm{m}^{2}$ of $200 \mathrm{MeV}$. Viscous flow leads to stress relaxation in subregion $1^{\prime}$. The stress field of 1 has a finite range into the unirradiated matrix $2^{\prime}$. Compressive stress in 1 is balanced by in-plane tensile stress in 3 . 
to region 1 reveals a transition region (region $2^{\prime}$ in Fig. 1) of about $20 \mu \mathrm{m}$ where the pore diameter gradually decreases to less than half of its value in region 2 near the borderline between regions $2^{\prime}$ and 1 (see Fig. 2). At the rim, this borderline is clearly visible as a step of about $2 \mu \mathrm{m}$ in depth. In region 1 , only very small etch pits with diameters of about $0.3 \mu \mathrm{m}$ were found. In regions $1^{\prime}$ the diameter of the etch pits increases gradually from $0.3 \mu \mathrm{m}$ to about $1 \mu \mathrm{m}$ at the free edges. Simultaneously, the borderline step separating regions $1^{\prime}$ and $2^{\prime}$ fades away (Fig. 3). It should be mentioned that similar observations were made when studying tracks of $720 \mathrm{MeV}$ Xe instead of $2.4 \mathrm{GeV} \mathrm{Pb}$ ions.

These experimental findings, particularly the changes of etchability observed in the transition regions $1^{\prime}$ and $2^{\prime}$, cannot be rationalized in terms of tracks solely characterized by structural defects but are in full accordance with the model idea that tracks form strained elastic inclusions. According to the model, ions penetrating the preirradiated region 1 cannot form well etchable tracks since there, the defect concentration is already close to its maximum and the compressive in-plane stress compensates shear stresses induced by local thermal expansion thus preventing shear stress relaxation and the freezing-in of a strain increment. With decreasing magnitude of the compressive stress, this suppression of track formation is released which explains the appearance of etch pits of increasing size in regions $1^{\prime}$ and the change of the size of etch pits in regions $2^{\prime}$. Close to the borderline between regions 1 and $2^{\prime}$ track etching is expected to be solely due to the increased defect concentration in the tracks.

We have made an attempt to analyze our experimental results quantitatively on the basis of the thermoelastic

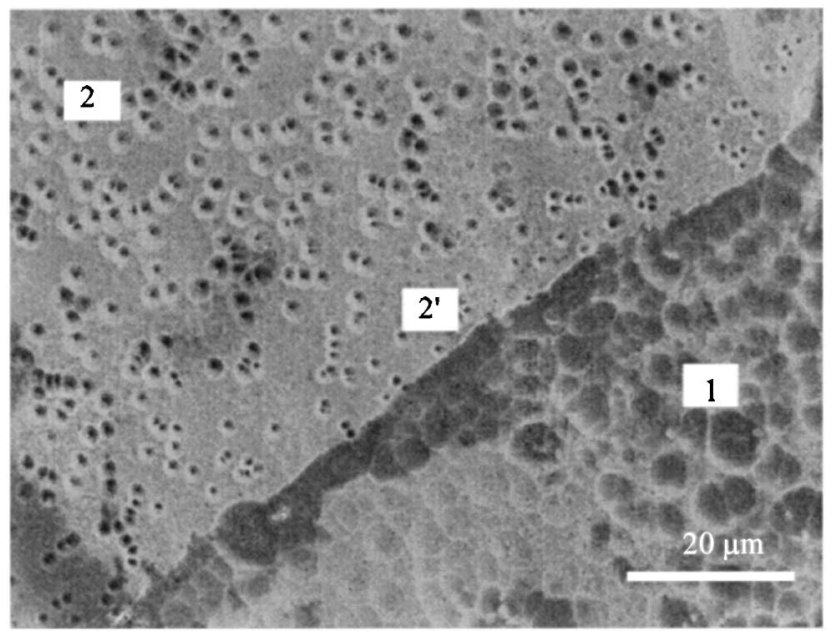

FIG. 2. Scanning electron micrograph of etched tracks in stressed and unstressed regions. In the stress-free region 2 (upper left zone) regular etch pits of about $3 \mu \mathrm{m}$ can be observed. In a transition regime $2^{\prime}$, the size of the pores decreases, while in the stressed region 1 (lower right zone) the pores are too small $(\sim 300 \mathrm{~nm})$ to be identified at this magnification. model and to check for data consistency. Taking into account that all differences in chemical potential changes per atom to be considered here are significantly smaller than $k T$, we may linearize the expressions for etching velocities with respect to these differences. Neglecting interfacial effects, we may then relate ratios of two such differences to ratios of two etching velocities, which can be deduced from our observations. Thus, for instance, we may write

$$
\delta \mu_{t} / \Delta \mu_{b}=v_{t} / v_{b}-1,
$$

where $v_{t}$ and $v_{b}$ are the etching velocities along a track and on the surface of the surrounding material, respectively. The relevant expressions provided by the thermoelastic model [19] yield 0.8 and $1.2 \mathrm{meV} /$ atom for the elastic contributions $\delta \mu_{t 2}^{\mathrm{el}}$ and $\delta \mu_{b 1}^{\mathrm{el}}$ to the chemical potential changes induced by individual tracks in region 2 and by the preirradiation in region 1 , respectively. These values may be used as references in estimating the other contributions. Since we have more experimental data than available theoretical parameters our analysis can be used as a consistency check. Thus, within the limited experimental accuracy, various parameters of the observed etch pits (average opening angle, diameter in region 2, diameter at the free etch of region $1^{\prime}$, and diameter and step height at the border between regions $2^{\prime}$ and 1) are consistent with the following values: $\Delta \mu_{b 2} \approx 2 \mathrm{meV} /$ atom for the difference in the chemical potential between the etchant and the virgin material of region 2 , and $\delta \mu_{t}^{\mathrm{def}} \approx \delta \mu_{b 1}^{\mathrm{def}} \approx 1 \mathrm{meV} /$ atom for the defect contributions to the chemical potential changes in tracks and in region 1.

The qualitative and semiquantitative agreement between model predictions and experimental observations demonstrates, first of all, the importance of the elastic contribution to the excess chemical potential driving preferential track etching in amorphous materials, and it confirms, beyond this, the thermal spike origin of the elastic strain

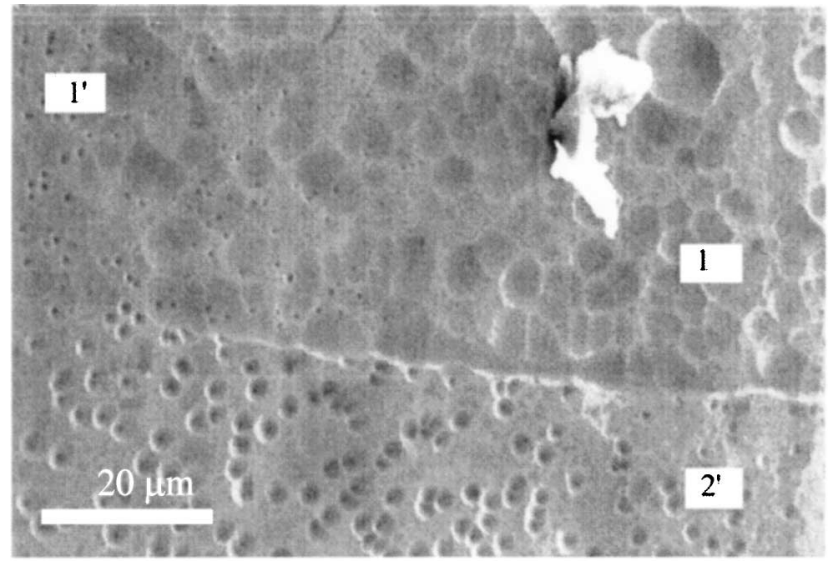

FIG. 3. Scanning electron micrograph of area close to the free edge of the sample (region $1^{\prime}$ ). The size of the etch pits increases as the position of the pores is closer to the outer edge (left vertical). 
in the tracks. This evidence for the validity of the thermal spike concept is further supported by the good agreement between model predictions and observed anisotropic growth data, particularly concerning the correlation of the growth rate with the thermal expansion coefficient of the material [18-20]. Based on our etching experiments alone, however, an unambiguous decision between a "Coulomb explosion" mechanism [2,22] and a thermal spike mechanism for explaining the stress/strain state of ion tracks is less straightforward. A possible distinctive criterion is the stopping power dependence of the stress effect on track formation. We discuss this briefly on the basis of some simple scaling arguments.

In the thermal spike model [17-20], creep and growth rates are proportional to the electronic stopping power $S_{e}$ via the linear dependence of the size of the fluid spike region on $S_{e}$ whereas the stress/strain within the tracks and correspondingly the saturation stress at which creep and growth compensate each other are independent of $S_{e}$ [19]. At this "zero creep stress," the formation of etchable tracks is suppressed independently of $S_{e}$ as confirmed by the similarity of our observations for Xe ions with $S_{e}=37 \mathrm{keV} / \mathrm{nm}$ and $\mathrm{Pb}$ ions with $S_{e}=55 \mathrm{keV} / \mathrm{nm}$.

The situation is less clear but certainly different in the Coulomb explosion model. According to Lesueur and Dunlop [22], the energy deposited per unit length into the Coulomb explosion spike is proportional to $S_{e}^{4}$. On the other hand, this energy scales with $\varepsilon_{\max }^{2} R^{2}$, where $\varepsilon_{\max }$ is the maximum strain in the initial phase of the Coulomb explosion and $R$ is the spatial scale of the ionized region. From both, one may conclude that $\varepsilon_{\max } \sim S_{e}^{2} / R$. Since the dependence of $R$ on $S_{e}$ is weaker than linear, the dependence of $\varepsilon_{\max }$ on $S_{e}$ is at least linear. Assuming that the material yields above a certain critical strain $\varepsilon^{*}$, we expect the local plastic strain and correspondingly the elastic strain in the track after a calming down of the Coulomb explosion to behave as $\varepsilon_{\mathrm{el}, \mathrm{pl}} \sim\left(\varepsilon_{\max }-\varepsilon^{*}\right)$. Accordingly, the stress suppressing track formation and anisotropic growth would clearly depend on $S_{e}$ and this particularly strongly close to the threshold $\left(\varepsilon_{\max } \approx \varepsilon^{*}\right)$. Under conditions where the formation of etchable tracks of our $\mathrm{Xe}$ ions is suppressed etching of our $\mathrm{Pb}$ ion tracks should be clearly possible-contrary to our findings. We therefore think that even the results of our etching experiments alone are in favor of the thermal spike model rather than the Coulomb explosion model.
*Corresponding author.

Electronic address: h.trinkaus@fz-juelich.de

[1] The present state of knowledge about track formation in solids is reflected in Proceedings of the Fourth International Conference on Swift Heavy Ions in Matter, Berlin, 1998 [Nucl. Instrum. Methods Phys. Res., Sect. B, 146 (1998)].

[2] R. L. Fleischer, P. B. Price, and R. M. Walker, Nuclear Tracks in Solids, Principles and Applications (University of California Press, Berkeley, 1975).

[3] R. Spohr, Ion Tracks and Microtechnology (Vieweg, Braunschweig, 1990).

[4] A. Sigrist and R. Balzer, Helv. Phys. Acta 50, 49 (1977).

[5] C. Trautmann, S. Andler, W. Brüchle, R. Spohr, and M. Toulemonde, Radiat. Eff. Defects Solids 126, 207 (1993).

[6] C. Trautmann, R. Spohr, and M. Toulemonde, Nucl. Instrum. Methods Phys. Res., Sect. B 83, 513 (1993).

[7] C. Trautmann, C. Dufour, E. Paumier, R. Spohr, and M. Toulemonde, Nucl. Instrum. Methods Phys. Res., Sect. B 107, 397 (1996).

[8] A. Audouard, R. Mamy, M. Toulemonde, G. Szenes, and L. Thomé, in Ref. [1], p. 217; Europhys. Lett. 40, 527 (1997).

[9] A. Dunlop, J. Henry, and G. Jaskierowicz, in Ref. [1], p. 222.

[10] S. Klaumünzer, Ming-Dong Hou, and G. Schumacher, Phys. Rev. Lett. 57, 850 (1986).

[11] Ming-Dong Hou, S. Klaumünzer, and G. Schumacher, Phys. Rev. B 41, 1144 (1990).

[12] A. Audouard, E. Balanzat, J. C. Jousset, D. Lesueur, and L. Thomé, J. Phys. Condens. Matter 5, 995 (1993).

[13] E. Snoeks, T. Weber, A. Cacciato, and A. Polman, J. Appl. Phys. 78, 4723 (1995).

[14] F. Garrido, A. Benyagoub, A. Chamberod, J.C. Dran, A. Dunlop, S. Klaumünzer, and L. Thomé, Nucl. Instrum. Methods Phys. Res., Sect. B 115, 430 (1996).

[15] T. Egami and D. Srolovitz, J. Phys. F 12, 2141 (1982).

[16] A. Audouard, J. Balogh, J. Dural, and J.C. Jousset, J. Non-Cryst. Solids 50, 71 (1982).

[17] H. Trinkaus, J. Nucl. Mater. 223, 196 (1995).

[18] H. Trinkaus and A. I. Ryazanov, Phys. Rev. Lett. 74, 5072 (1995).

[19] H. Trinkaus, Nucl. Instrum. Methods Phys. Res., Sect. B 107, 155 (1996).

[20] H. Trinkaus, Nucl. Instrum. Methods Phys. Res., Sect. B 146, 204 (1998).

[21] J. F. Ziegler, J. P. Biersack, and U. Littmark, The Stopping and Ranges of Ions in Solids (Pergamon Press, New York, 1985).

[22] D. Lesueur and A. Dunlop, Radiat. Eff. Defects Solids 126, 163 (1993). 